\title{
Learning to like disgust: neuronal correlates of counterconditioning
}

\section{Jan Schweckendiek ${ }^{1 *}$, Tim Klucken ${ }^{1}$, Christian J. Merz ${ }^{1,2}$, Sabine Kagerer ${ }^{1}$, Bertram Walter ${ }^{3}$, Dieter Vait/ ${ }^{3}$ and Rudolf Stark ${ }^{1,3}$}

${ }^{1}$ Department of Psychotherapy and Systems Neuroscience, Justus Liebig University Giessen, Giessen, Germany

${ }^{2}$ Department of Cognitive Psychology, Institute of Cognitive Neuroscience, Ruhr-University Bochum, Bochum, Germany

${ }^{3}$ Bender Institute of Neuroimaging, Justus Liebig University Giessen, Giessen, Germany

Edited by:

John J. Foxe, Albert Einstein College of Medicine, USA

Reviewed by:

Alexander J. Skolnick, Saint Joseph's University, USA

Suzanne Oosterwijk, University of

Amsterdam, Netherlands

Elizabeth A. Krusemark, University

of Wisconsin, USA

*Correspondence:

Jan Schweckendiek, Department of

Psychotherapy and Systems

Neuroscience, Justus Liebig

University Giessen,

Otto-Behaghel-Strasse $10 \mathrm{H}$,

35394 Giessen, Germany

e-mail: jan.h.schweckendiek@

psychol.uni-giessen.de
Converging lines of research suggest that exaggerated disgust responses play a crucial role in the development and maintenance of certain anxiety disorders. One strategy that might effectively alter disgust responses is counterconditioning. In this study, we used functional magnetic resonance imaging (fMRI) to examine if the neuronal bases of disgust responses are altered through a counterconditioning procedure. One disgust picture (conditioned stimulus: $\mathrm{CS}+{ }_{\text {disg }}$ ) announced a monetary reward, while a second disgust picture (CS-disg) was never paired with the reward. Two neutral control pictures $\left(\mathrm{CS}+_{\text {con }} / \mathrm{CS}_{-}{ }_{\text {con }}\right)$ were conditioned in the same manner. Analyses of evaluative conditioning showed that both $\mathrm{CS}+$ were rated significantly more positive after conditioning as compared to the corresponding CS-. Thereby, the CS+disg and the $\mathrm{CS}+{ }_{\text {con }}$ received an equal increase in valence ratings. Regarding the $\mathrm{fMRI}$ data, ANOVA results showed main effects of the conditioning procedure (i.e., CS+ vs. CS-) in the dorsal anterior cingulate cortex. Further, main effects of the picture category (disgust vs. control) were found in the bilateral insula and the orbitofrontal cortex. No interaction effects were detected. In conclusion, the results imply that learning and anticipation of reward was not significantly influenced by the disgust content of the CS pictures. This suggests that the affect induced by the disgust pictures and the affect created by the anticipation of reward may not influence the processing of each other.

Keywords: counterconditioning, classical conditioning, evaluative conditioning, fMRI, disgust, reward learning

\section{INTRODUCTION}

A growing line of evidence suggests that the emotion disgust plays an important role in the etiology and maintenance of psychiatric disorders like obsessive-compulsive disorder, specific phobias, eating disorders, and even post-traumatic stress disorder (Olatunji et al., 2010; Mason and Richardson, 2012). The emotion disgust has unique features and has been shown to be very resistant to extinction (Rozin and Fallon, 1987; Olatunji et al., 2010; Mason and Richardson, 2012). This may in part explain the difficulties in the treatment of these disorders (Mason and Richardson, 2012) with exposure therapy, which is based on extinction (e.g., McNally, 2007). Insight into the neuronal circuitry underlying the alteration of disgust responses can be used to improve treatment strategies. In the present study, we investigated if subjective and hemodynamic disgust responses are altered through a counterconditioning procedure.

Although counterconditioning has been examined in some detail in animals (e.g., Dickinson and Pearce, 1977; Bouton, 2004), human studies are sparse. This is surprising, since many influential theories of reinforcement learning make explicit predictions for counterconditioning (e.g., Dickinson and Pearce, 1977 see Daw et al., 2002). Counterconditioning describes the process in which a CS is first paired with one unconditioned stimulus (UCS) and then paired with another
UCS of incompatible affective value in a second step (Bouton, 2004). However, in some experimental designs counterconditioning refers merely to the pairing of stimuli of opposing valence (cf. Jong et al., 2000). This was also the case in the present study, in which disgust inducing stimuli were paired with an appetitive reward stimulus. One recent study was able to show that counterconditioning of conditioned disgust-related evaluative responses was more effective compared to extinction as measured by pleasantness ratings and an affective priming task (Kerkhof et al., 2011). Moreover, counterconditioning has been found to improve exposure therapy in spider phobics with regard to valence and fear ratings as well as heart rate changes (Eifert et al., 1988 but see Jong et al., 2000). Although both studies did not directly measure disgust ratings, the results suggest that counterconditioning can modify evaluative responding to disgust stimuli, at least in terms of valence. However, no study to date has examined the underlying neuronal mechanisms.

Given that counterconditioning is able to change responding to disgust stimuli, this should result in an alteration of brain activity in areas that have been associated with disgust processing. The insula plays a central role in disgust processing and the recognition of disgust from facial expressions. Enhanced insula reactions were observed in response to disgust inducing pictures and video clips in a variety of studies (e.g., Schienle et al., 2002b; Wright 
et al., 2004; Caseras et al., 2007; Jabbi et al., 2008; Schäfer et al., 2009). Moreover, insula activity is correlated with the subjective experience of disgust (Fitzgerald et al., 2004; Stark et al., 2007) and the personality trait disgust sensitivity (Calder et al., 2001; Schienle et al., 2003; Stark et al., 2005; Caseras et al., 2007; Schäfer et al., 2009; Olatunji et al., 2010; Klucken et al., 2012a). In addition to the insula, converging evidence points to the amygdala, the orbitofrontal cortex (OFC), and the dorsal striatum as important structures in disgust processing (Calder et al., 2001; Phan et al., 2004; Vytal and Hamann, 2010).

Moreover, because counterconditioning of disgust stimuli entails aspects of reward learning and anticipation, it could also affect areas related to these processes. Studies in animals and humans have implicated the ventral striatum [especially the nucleus accumbens (NAcc)], the OFC, the amygdala, the dorsal anterior cingulate cortex (dACC), and the insula (MartinSoelch et al., 2007; Klucken et al., 2009b; Haber and Knutson, 2010; Klucken et al., 2012b). Activity of the NAcc and the OFC has been reported to shift from the onset of the UCS to the onset of the CS as the occurrence of the UCS becomes more predictable during the course of conditioning (Schultz, 1997; McClure et al., 2003; O'Doherty et al., 2003). The amygdala has been consistently implicated in animal studies of reward learning and anticipation (Haber and Knutson, 2010), however, only few human studies have reported an involvement of the amygdala (e.g., Gottfried et al., 2002). In addition, studies using monetary reward as UCS have reported dACC and insula activation (Kirsch et al., 2003; Cox et al., 2005). The dACC has not only been consistently implicated in reward-related learning and anticipation, but also in tasks that require error detection, response override, and other forms of conflict including emotional conflict (for review see Botvinick, 2007; Carter and van Veen, 2007; Taylor et al., 2007). Moreover, it has been proposed that the dACC and the insula form the core of a salience network, which is activated in response to important environmental stimuli (Menon and Uddin, 2010).

In the present study, we tested whether activity of the mentioned brain regions is altered by a counterconditioning procedure. To this end, we designed a novel paradigm using a classical conditioning approach. In a differential conditioning design, one disgust picture $\left(\mathrm{CS}+_{\text {disg }}\right)$ predicted a monetary reward (UCS), while a second disgust picture $\left(\mathrm{CS}-{ }_{\text {disg }}\right)$ was never paired with the reward. A second pair of neutral control pictures $\left(\mathrm{CS}+_{\text {con }} / \mathrm{CS}-_{\text {con }}\right)$ served as a control condition and was differentially conditioned in the same manner, again using monetary reward as UCS. Using this 2 (CS-emotion: disgust vs. control) $\times 2$ (reward learning: $\mathrm{CS}+$ vs. $\mathrm{CS}-$ ) factorial design allowed us to investigate the effect of the counterconditioning procedure while controlling for mere effects of the emotional content of the pictures and the effects of reward learning and anticipation (i.e., of conditioning). In accordance with previous studies, we hypothesized that the counterconditioning procedure would shift subjective valence ratings of the $\mathrm{CS}+_{\text {disg }}$ in the positive direction. Regarding hemodynamic responses, we expected the CS+disg to elicit altered activity of structures related to the processing of disgust responding and of structures related to reward learning and anticipation as compared to the CS- disg. In detail, we expected enhanced responses of the AACC, the insula, the NAcc, the amygdala, and the OFC as a correlate of the counterconditioning procedure.

\section{METHODS AND MATERIALS SUBJECTS}

Thirty-two healthy (16 female, 16 male) subjects were recruited from campus advertisements; four subjects were excluded from analyses because of extensive head movement, drowsiness during scanning (two subjects), and an extremely low disgust sensitivity score (i.e., $>2$ standard deviations below the group mean; Schienle et al., 2002a) leaving 28 subjects in the final sample (12 male, 16 female; $M_{\text {age }}=25.93 ; S D_{\text {age }}=3.22$ ). All subjects were students at the Justus Liebig University Giessen, right-handed, and had normal or corrected-to-normal vision. No subject had ever received psychotropic medication or psychotherapeutic treatment. Participants were informed about the procedure in general and gave written informed consent. All experimental procedures were in accordance with the Declaration of Helsinki and were approved by the local ethics committee of the Institute for Psychology and Sports Science at the Justus Liebig University Giessen.

\section{STIMULI}

Two pictures of disgust scenes (dirty toilets) and two pictures of household items (a dish and a stool) served as CS in the experimental condition. Two pictures were taken from the International Affective Pictures System (Lang et al., 2008; picture numbers: 7006, 9300), the other two were collected by the authors. All pictures had been successfully used in previous studies (Stark et al., $2004,2007)$. An amount of $0.50 €$ was used as UCS, which was represented by a cartoon drawing of coin stacks collected by the authors. Pictures were comparable with regard to complexity as far as possible in order to prevent confounding effects. Stimuli were projected onto a screen at the end of the scanner (visual field $=18^{\circ}$ ) using an LCD projector (EPSON EMP-7250) and were viewed through a mirror mounted on the head coil.

\section{PROCEDURE}

Subjects were instructed that they would be exposed to emotionally disgust scenes and pictures of everyday items. Further, subjects were told that they would receive $15 €$ for participation and an additional amount of $0.50 €$ for each time they saw the picture of the coin stacks at the end of the experiment and that they didn't have to do anything to obtain the money. Moreover, subjects were instructed to look at the pictures and to pay attention to possible relationships between the monetary reward and the other pictures presented during the experiment (cf. Schiller et al., 2008; Raes et al., 2009; Schweckendiek et al., 2011; Klucken et al., 2012a).

The classical conditioning design was adopted from previous studies using pictures as UCS (e.g., Klucken et al., 2009a,b; Schweckendiek et al., 2011). During the experiment subjects passively viewed the images while hemodynamic responses were recorded. Except for the subjective ratings (see below), no other behavioral measures were collected. The experiment consisted of a habituation phase, a conditioning phase, and an extinction 
phase. During the habituation phase each of the four CS pictures was presented 10 times. One trial consisted of the presentation of a CS picture for $3 \mathrm{~s}$ followed by the inter trial interval (ITI), which ranged from 3 to $8 \mathrm{~s}$ (see below). During the conditioning phase each CS was presented 16 times for $8 \mathrm{~s}$. One disgust $\left(\mathrm{CS}+_{\text {disg }}\right)$ and one neutral picture $\left(\mathrm{CS}+{ }_{\text {con }}\right)$ were followed by the picture of coin stacks (UCS) that represented the gain of $0.50 €$ for $3 \mathrm{~s}$ with $100 \%$ reinforcement with no delay, while the remaining disgust $\left(\mathrm{CS}-_{\text {disg }}\right)$ and neutral $\left(\mathrm{CS}-_{\text {con }}\right)$ pictures were never followed by the UCS. The ITI again ranged from 3 to $8 \mathrm{~s}$ (see below). During the extinction phase, all CS were again presented 10 times each for $3 \mathrm{~s}$. One trial again consisted of the presentation of a CS picture and the ITI, which ranged from 3 to $8 \mathrm{~s}$ (see below). In total, subjects were exposed to each picture for $30 \mathrm{~s}(10 \times 3 \mathrm{~s})$ during the habituation and for $128 \mathrm{~s}(16 \times 8 \mathrm{~s})$ during the conditioning phase. The short extinction phase is part of another project and will not be discussed here in detail. Only data from the habituation and the conditioning phase are reported in this manuscript.

Stimulus allocation as CS+ and CS- was counterbalanced between participants. The ITI was equally distributed between 3 and 8 s. A small fixation cross was presented at the center of the screen during the ITI. ITIs were calculated to contain equally distributed stimulus-onset-asynchronies (ranging from 0 to $2.5 \mathrm{~s}$ ) in order to optimize signal acquisition for the wholebrain. Stimuli were presented in a pseudo-randomized order with the restrictions: (1) no more than two consecutive presentations of the same CS, (2) no more than two consecutive presentations of the same CS-type (i.e., CS+/CS-), (3) no more than two consecutive presentations of the same picture category (i.e., disgust/control), and (4) an equal quantity of each CS within the first and the second half of the conditioning phase. Throughout the experiment an MRI-compatible video camera was used to insure that subjects watched the stimuli. After the experiment, participants filled out the Questionnaire for the Assessment of Disgust Sensitivity (QADS; Schienle et al., 2002a) assessing individual proneness to disgust. The questionnaire was designed based on the questionnaire by Haidt et al. (1994) and describes 37 situations, which have to be judged on a five point scale regarding their ability to induce disgust. The questionnaire consists of five different subscales: (1) death/deformation (2) body secretion (3) spoilage/decay (4) poor hygiene (5) oral rejection. Cronbach's $\alpha$ of the total scale is 0.90 with the subscales varying between 0.69 and 0.85 .

\section{SUBJECTIVE RATINGS OF THE CS}

In addition to the neuroimaging data, subjective ratings of the stimuli were collected. Before the habituation phase and after the conditioning phase subjects rated valence, arousal, and disgust for each of the four CS $\left(\mathrm{CS}+{ }_{\text {disg }}\right.$; $\mathrm{CS}-_{\text {disg }}$; $\left.\mathrm{CS}+_{\text {con }} ; \mathrm{CS}-_{\text {con }}\right)$ on a nine-point Likert scale ranging from 1 ("very unpleasant"; "calm and relaxed"; "not disgusting at all") to 9 ("very pleasant"; "very arousing"; "very disgusting"). Arousal and disgust ratings were collected on an exploratory basis. The measurement of subjective ratings before and after conditioning ensured that differences in the ratings were due to the counterconditioning procedure, while controlling for pre-existing differences and effects of the repeated presentation of the stimuli. The assessment of subjective ratings before the habituation phase ensured a relatively unbiased evaluation of the stimuli. Statistical analyses of the ratings were performed by means of a $2 \times 2$ $\times 2$ ANOVA with the within-subject factors "reward learning" (CS+ / CS-), "phase" (habituation phase, conditioning phase) and "CS-emotion" (disgust/control) as implemented in SPSS 19 (IBM Corporation, Armonk, NY, USA) separately for each of the three rating dimensions (valence, arousal, disgust).

\section{MAGNETIC RESONANCE IMAGING}

Functional and anatomical scans were obtained using a $1.5 \mathrm{~T}$ whole-body tomography (Siemens Symphony) with a standard head coil. Structural image acquisition consisted of 160 T1weighted sagittal images (MPRage, $1 \mathrm{~mm}$ slice thickness). A gradient echo field map was acquired before the functional image acquisition to obtain information for unwarping $\mathrm{B}_{0}$ distortions. For functional imaging a total of 832 volumes were recorded using a T2*-weighted gradient echo-planar imaging sequence (EPI) with 25 slices covering the whole-brain (slice thickness $=5 \mathrm{~mm}$; gap $=1 \mathrm{~mm}$; descending slice order; $T A=100 \mathrm{~ms} ; T E=55 \mathrm{~ms}$; $T R=2.5 \mathrm{~s}$; flip angle $=90^{\circ}$; field of view $=192 \times 192 \mathrm{~mm}$; matrix size $=64 \times 64$ pixel). The orientation of the axial slices was tilted $30^{\circ}$ to the AC-PC line to keep susceptibility artifacts in the ventromedial parts of the frontal cortex to a minimum (cf. Deichmann et al., 2003; Weiskopf et al., 2006). Functional data were analyzed for outlying volumes using a distribution free approach for skewed data: outlier detection was based on a comparison of each volume with its two neighbors in a motion corrected time series. This was done by calculating the mean squared differences to the previous and the next volume. The smaller difference was used as deviation score for each volume. The scores were thresholded using the method of Hubert and van der Veeken (2008). Each resulting outlying volume was later modeled within the general linear model (GLM) with a covariate of no interest.

Preprocessing and statistical analyses were performed using Statistical Parametric Mapping (SPM8, Wellcome Department of Cognitive Neurology, London, UK; 2009) implemented in Matlab R2007b (Mathworks Inc., Sherborn, MA). Preprocessing of functional data included unwarping and realignment to the first volume (b-spline interpolation), slice time correction, normalization to the standard space of the Montreal Neurological Institute brain (MNI-brain) and smoothing with an isotropic three dimensional Gaussian kernel with a full-width-at-half-maximum (FWHM) of $9 \mathrm{~mm}$.

Following experimental conditions were modeled in the general linear model for each subject: CS+ disg (paired disgust CS),

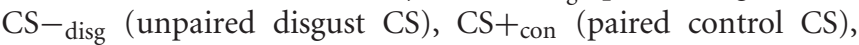
$\mathrm{CS}-{ }_{\text {con }}$ (unpaired control CS) separately for the different phases of the experiment, UCS and non-UCS (i.e., the time after the CScorresponding to the time of the UCS presentation after the CS+; Klucken et al., 2009a,b; Merz et al., 2010). In addition, the rating phases were modeled as nuisance regressors. The experimental conditions were modeled by stick functions convolved with the canonical hemodynamic response function. The six movement parameters obtained by the realignment procedure as well as the 
outlying volumes were introduced as covariates in the model. Additionally, a high pass filter (time constant $=180 \mathrm{~s}$ ) was implemented using discrete cosine functions regressors. The subject level models were estimated after pre-whitening.

Beta-estimates of each regressor were calculated for each individual and were introduced as dependant variables to the second level random effects group analyses. Because individual disgust sensitivity is known to influence neuronal activity in response to disgust stimuli (Calder et al., 2001; Schienle et al., 2003; Stark et al., 2005; Caseras et al., 2007; Schäfer et al., 2009; Olatunji et al., 2010; Klucken et al., 2012a,b), DS scores were introduced as covariate of no interest to all contrasts involving the disgust pictures. DS scores were also correlated (voxel-wise simple regression) with the contrast $\mathrm{CS}+{ }_{\text {disg }}$ vs. $\mathrm{CS}-{ }_{\text {disg. }}$. As a manipulation check, we first compared the UCS to the non-UCS in the conditioning phase and, in addition, the disgust to the control pictures in the habituation phase using paired $t$-tests.

To analyze main and interaction effects during the conditioning phase a 2 ("CS-emotion": disgust vs. control) $\times 2$ ("reward learning": CS+ vs. CS-) full factorial model (Penny and Henson, 2007) was used in order to avoid potentially biased Type I errors in second level analyses due to the use of pooled errors (Boik, 1981; Barcikowski and Robey, 1984). Further we compared CS+ $+_{\text {disg }}$ vs. $\mathrm{CS}-_{\text {disg }}$, as well as $\mathrm{CS}+_{\text {con }}$ vs. $\mathrm{CS}-_{\text {con }}$ using one-sample $t$-tests. Moreover, in order to link the hemodynamic responses to evaluative conditioning, we correlated (voxel-wise simple regression) the mean differential valence scores with the contrasts $\mathrm{CS}+{ }_{\text {disg }}$ vs. $\mathrm{CS}-$ disg and $\mathrm{CS}+{ }_{\text {con }}$ vs. CS - con .

Within all models, we first performed explorative whole-brain analyses $\left(p_{\mathrm{FWE}}<0.05\right.$ corrected for the whole-brain; Worsley, 2007). The next step was to test our a priori regions of interest (ROI) using the small volume correction feature of SPM ( $p_{\text {FWE }}<0.05$ corrected for search volume; (Worsley, 2007); cluster forming threshold: $p_{\text {uncorr }}<0.001, k>5$ ). The ROI analyses were performed for the following structures: insula, dACC, NAcc, amygdala, OFC, and dorsal striatum. All masks were created from the probabilistic Harvard-Oxford Cortical and Subcortical
Atlases (included in FSLView version 3.1; http://www.fmrib.ox. ac.uk/fsl/; cf. Schweckendiek et al., 2011). Anatomical labeling of the exploratory whole-brain analyses was also performed using the Harvard-Oxford Cortical and Subcortical Atlases. The significance threshold was set to $\alpha=0.05$ corrected for multiple testing using family-wise-error correction as implemented in SPM8

\section{RESULTS}

\section{ANALYSES OF SUBJECTIVE RATINGS}

Subjective valence, arousal, and disgust ratings were analyzed separately using a 2 ("reward learning": CS+ vs. CS-) $\times 2$ ("phase": habituation vs. conditioning) $\times 2$ ("CS-emotion": disgust vs. control) ANOVA.

Regarding the analyses of the valence ratings, the three-way interaction did not reach the significance level $(p>0.15)$. A significant two-way interaction effect of the factors "phase" $x$ "reward learning" $\left[F_{(1,27)}=6.44 ; p=0.017\right]$ was observed. Both $\mathrm{CS}+$ (i.e., $\mathrm{CS}+_{\text {disg }}$ and $\left.\mathrm{CS}+_{\text {con }}\right)$ were rated more positively after conditioning than before the habituation phase as compared to their corresponding CS- (see Figure 1). Moreover, a highly significant main effect of "CS-emotion" was found $\left[F_{(1,27)}=65.56\right.$; $p<0.001]$ : the disgust pictures were rated as significantly more unpleasant as compared to the control pictures (see Figure 1). In addition, a significant main effect of the factor "phase" was observed $\left[F_{(1,27)}=7.49 ; p<0.011\right]$, with overall more positive valence ratings after conditioning compared to before the habituation phase.

Post hoc analyses (with Bonferroni correction) of the valence ratings confirmed that before the habituation phase, there was no significant difference in valence ratings between $\mathrm{CS}+$ disg and $\mathrm{CS}-_{\text {disg }}(p>0.2)$. Likewise, $\mathrm{CS}+{ }_{\text {con }}$ and $\mathrm{CS}-_{\text {con }}$ were not rated differently $(p>0.2)$ before the habituation phase. After conditioning, significant differences emerged for the comparison $\mathrm{CS}+_{\text {disg }}$ vs. $\mathrm{CS}-{ }_{\text {disg }}\left[t_{(27)}=3.17 ; p=0.004\right]$ and the comparison $\mathrm{CS}+{ }_{\text {con }}$ vs. CS $-_{\text {con }}\left[t_{(27)}=3.35 ; p=0.002\right]$. Both CS+ were rated more positively as compared to the corresponding CS-.

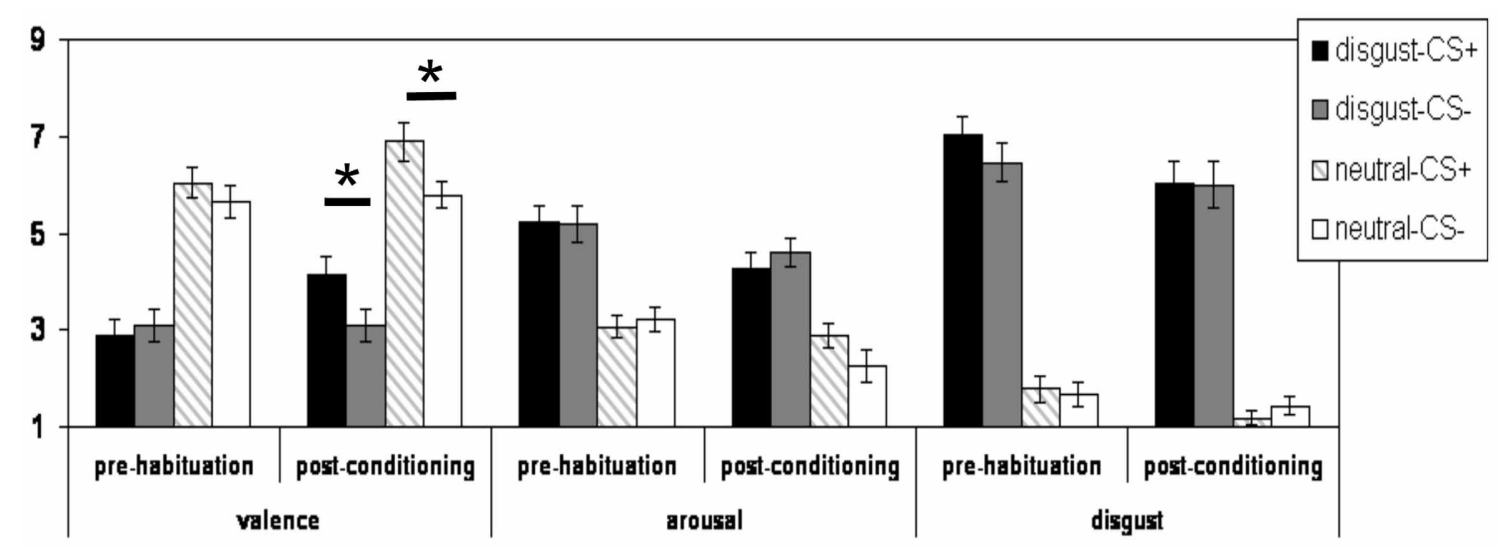

FIGURE 1 | Mean subjective valence, arousal, and disgust ratings (and standard errors of the mean) of for the CS+disg, the CS $-_{\text {disg }}$, the CS+con, and the $\mathrm{CS}_{-}$con before the habituation phase and after the conditioning phase. ${ }^{*}$ indicates $p<0.05$. 
Regarding the analyses of the arousal ratings, no significant interaction effects were observed (all $p>0.05$ ). Yet, a significant main effect of the factor "CS-emotion" was detected $\left[F_{(1,27)}=\right.$ 37.72; $p<0.001$ ]: the disgust pictures were rated as significantly higher arousing as compared to the control pictures. Moreover, a significant main effect of the factor "phase" was observed $\left[F_{(1,27)}=7.93 ; p=0.009\right]$ : overall, arousal ratings were lower after the conditioning as compared to before the habituation phase.

Regarding the analyses of the disgust ratings, no significant interaction effects were observed (all $p>0.10$ ). We again observed a significant main effect of the factor "CS-emotion" $\left[F_{(1,27)}=161.91 ; p<0.001\right]$ : as expected, the disgust CS received higher disgust ratings. Moreover, we observed a significant main effect of the factor "phase" $\left[F_{(1,27)}=7.12 ; p<\right.$ $0.012]$.

Next, we correlated the differential rating scores of the comparison $\mathrm{CS}+{ }_{\text {disg }}$ to $\mathrm{CS}-$ disg $_{\text {di.e., }}\left(\mathrm{CS}+{ }_{\text {disg }} \text { to } \mathrm{CS}-{ }_{\text {disg }}\right)_{\text {pre-habituation }}$ $\left.\left(\mathrm{CS}+{ }_{\text {disg }} \text { to } \mathrm{CS}-{ }_{\text {disg }}\right)_{\text {post-conditioning }}\right]$ with the disgust sensitivity scores. We found a significant positive correlation of disgust sensitivity with the differential arousal ratings $(r=0.44 ; p=0.021)$.
The other ratings scales were not significantly correlated with the disgust sensitivity scores $(p>0.40)$.

\section{fMRI}

\section{Manipulation check}

As a manipulation check, we first analyzed the contrast UCS > non-UCS in the conditioning phase. As expected, the contrast UCS > non-UCS revealed strong whole-brain as well as ROIactivation (e.g., bilaterally in the NAcc). Statistical parameters and coordinates of the significant results of the exploratory wholebrain as well as the ROI analyses for this contrast can be found in Table 1.

Next, as a second manipulation check, we analyzed the contrast of the two disgust vs. the two control pictures in the habituation phase. The exploratory whole-brain analyses revealed stronger activity to the disgust pictures. Two bilateral clusters of activation, both ranging from primary visual cortex areas into the posterior fusiform gyrus peaking in the left occipital pole $(x=$ $\left.-24, y=-94, z=-2 ; t=13.47 ; k=893 ; p_{\mathrm{FWE}}<0.001\right)$ and the right lingual gyrus $(x=27, y=-49, z=-8 ; t=12.53 ; k=$ 834; $\left.p_{\text {FWE }}<0.001\right)$, respectively, were observed. Further, ROI

Table 1 | Correction volume, structures, cluster sizes $(k)$, coordinates of peak voxels, $t$-values, and $\boldsymbol{p}_{\text {corr }}$-values (FWE correction) for the contrast UCS > non-UCS (from the conditioning phase).

\begin{tabular}{|c|c|c|c|c|c|c|c|c|}
\hline \multicolumn{9}{|c|}{ Contrast: UCS > non-UCS } \\
\hline Whole-brain & Occipital fusiform gyrus & $\mathrm{R}$ & 5745 & 30 & -76 & -8 & 20.05 & $<0.001$ \\
\hline & Precentral gyrus & $\mathrm{R}$ & 406 & 42 & 8 & 28 & 11.13 & $<0.001$ \\
\hline & Frontal orbital cortex & $\mathrm{R}$ & 300 & 33 & 26 & -5 & 10.68 & $<0.001$ \\
\hline & Thalamus & $\mathrm{R}$ & 242 & 21 & -31 & 1 & 10.45 & $<0.001$ \\
\hline & Frontal orbital cortex & $L$ & 196 & -30 & 23 & -8 & 8.71 & $<0.001$ \\
\hline & Frontal pole & $\mathrm{R}$ & 72 & 42 & 59 & 1 & 7.98 & $<0.001$ \\
\hline & Posterior cingulate gyrus & $L$ & 53 & -3 & -25 & 28 & 7.89 & 0.001 \\
\hline & Superior frontal gyrus & $L$ & 13 & -18 & 17 & 67 & 7.50 & 0.002 \\
\hline & Frontal pole & $\mathrm{R}$ & 75 & 51 & 47 & 19 & 7.03 & 0.005 \\
\hline \multirow[t]{9}{*}{$\mathrm{ROI}$} & OFC & $\mathrm{R}$ & 315 & 33 & 26 & -5 & 10.68 & $<0.001$ \\
\hline & Insula & $\mathrm{R}$ & 147 & 33 & 26 & -2 & 10.65 & $<0.001$ \\
\hline & OFC & $\mathrm{L}$ & 293 & -30 & 23 & -8 & 8.71 & $<0.001$ \\
\hline & Insula & $L$ & 156 & -30 & 23 & -2 & 8.70 & $<0.001$ \\
\hline & ACC & $\mathrm{R}$ & 293 & 3 & 23 & 34 & 7.74 & $<0.001$ \\
\hline & NAcc & $L$ & 22 & -12 & 11 & -11 & 5.13 & $<0.001$ \\
\hline & Amygdala & $L$ & 16 & -21 & -4 & -11 & 4.72 & 0.002 \\
\hline & Amygdala & $\mathrm{R}$ & 5 & 30 & 2 & -17 & 3.90 & 0.014 \\
\hline & NAcc & $\mathrm{R}$ & 3 & 12 & 14 & -5 & 3.64 & 0.009 \\
\hline
\end{tabular}

Labeling of the results from the exploratory whole-brain analyses was performed using the probabilistic Harvard-Oxford Cortical and Subcortical Atlases. The significance threshold was $p_{\text {corr }}<0.05$. The cluster forming threshold was $p_{\text {corr }}<0.05$ and $k \geq 5$ voxel for the whole-brain analyses and $p_{\text {uncorr }}<0.001$ and $k \geq 5$ for the ROI analyses. All coordinates are given in MNI space. 
analyses revealed significant bilateral OFC (right: $x=27, y=32$, $z=-14 ; t=5.29 ; p_{\mathrm{FWE}}=0.003$; left: $x=-33, y=23, z=-8$; $\left.t=4.88 ; p_{\mathrm{FWE}}=0.007\right)$, right amygdala $(x=24, y=-7, z=$ $\left.-14 ; t=4.93 ; p_{\mathrm{FWE}}=0.002\right)$, as well as left insula activation $\left(x=-33, y=-20, z=-5 ; t=5.08 ; p_{\mathrm{FWE}}=0.004\right)$.

\section{Analyses of conditioned responses}

Turning to the main analysis of hemodynamic activity during the conditioning phase, conditioned responses (CR) were analyzed using a 2 ("CS-emotion": disgust vs. control) $\times 2$ ("reward learning": CS+ vs. CS-) ANOVA. The exploratory whole-brain analyses did not yield a significant interaction effect or a significant main effect of reward learning. We found significant whole-brain main effects for the factor "CS-emotion" (i.e., disgust vs. control), which were similar to the results of the contrast disgust $>$ control in the habituation phase. Again, strong wholebrain effects were found in visual areas, with peaks in the occipital pole and in the thalamus (see Figure 2A and Table 2).

Next, we analyzed our a priori ROI. Again, no significant interaction effects were detected. Moreover, ROI analyses revealed a main effect of "reward learning" in the dACC (see Figure 2B and Table 2). Inspection of beta-estimates revealed that this was due to larger dACC activity in response to the two CS+ as compared to the two CS-. ROI analyses of the main effect "CS-emotion" showed significant effects bilaterally in the insula and in the right OFC (see Figure 2A). Assessment of beta-estimates confirmed that these effects were due to larger responses to the disgust than to the control pictures. All statistical parameters and MNIcoordinates regarding the results of the ANOVA can be found in Table 2.

Next, we analyzed following contrasts using paired $t$-tests: $\mathrm{CS}+{ }_{\text {disg }}>\mathrm{CS}-{ }_{\text {disg }}$ and $\mathrm{CS}+{ }_{\text {con }}>\mathrm{CS}-{ }_{\text {con }}$. The contrast $\mathrm{CS}+{ }_{\text {disg }}$ $>\mathrm{CS}-$ disg revealed no significant effects in the exploratory whole-brain analyses. The ROI analyses (see Figure 3A) in the same contrast showed significant activation in the dACC $(x=$ $\left.-6, y=-1, z=43 ; t=4.67 ; p_{\mathrm{FWE}}=0.014\right)$ and the left insula $\left(x=-33, y=11, z=-5 ; t=4.11 ; p_{\mathrm{FWE}}=0.031\right)$. Finally, in the contrast $\mathrm{CS}+{ }_{\text {con }}>\mathrm{CS}-_{\text {con }}$ exploratory whole-brain analyses again did not show significant effects. Subsequent ROI analyses (see Figure 3B) revealed significant differentiation in the right $\operatorname{NAcc}\left(x=12, y=11, z=-8 ; t=3.72 ; p_{\mathrm{FWE}}=0.007\right)$.

\section{CORRELATIONAL ANALYSES}

Regarding the correlational analyses, we found no significant results for the correlation of the hemodynamic activity in the contrast $\mathrm{CS}+_{\text {disg }}>\mathrm{CS}-_{\text {disg }}$ with the subjective valence ratings. Activity in the dACC exceeded the significance threshold only marginally $\left(x=-6, y=-1, z=37 ; t=4.03 ; p_{\mathrm{FWE}}=0.064\right.$; $k=8$ ). Interestingly, this was the same voxel which showed the main effect of conditioning in the ANOVA of conditioned responses.

Regarding the correlational analyses of the contrast $\mathrm{CS}+$ con $>$ $\mathrm{CS}-{ }_{\text {con }}$ with the subjective valence ratings, we did not find any significant results.

Regarding the correlational analyses of disgust sensitivity with the contrast $\mathrm{CS}+_{\text {disg }}>\mathrm{CS}-_{\text {disg }}$, we again did not observe significant findings.

\section{DISCUSSION}

In the present study, we investigated whether activity in brain areas that have been previously associated with disgust responses and/or to reward learning and anticipation are altered by a counterconditioning procedure. Clear evaluative conditioning effects were found with regard to the subjective valence ratings, however, no interaction effect was observed, i.e., the increase in valence was the same for the $\mathrm{CS}+_{\text {disg }}$ and the $\mathrm{CS}+_{\text {con }}$.

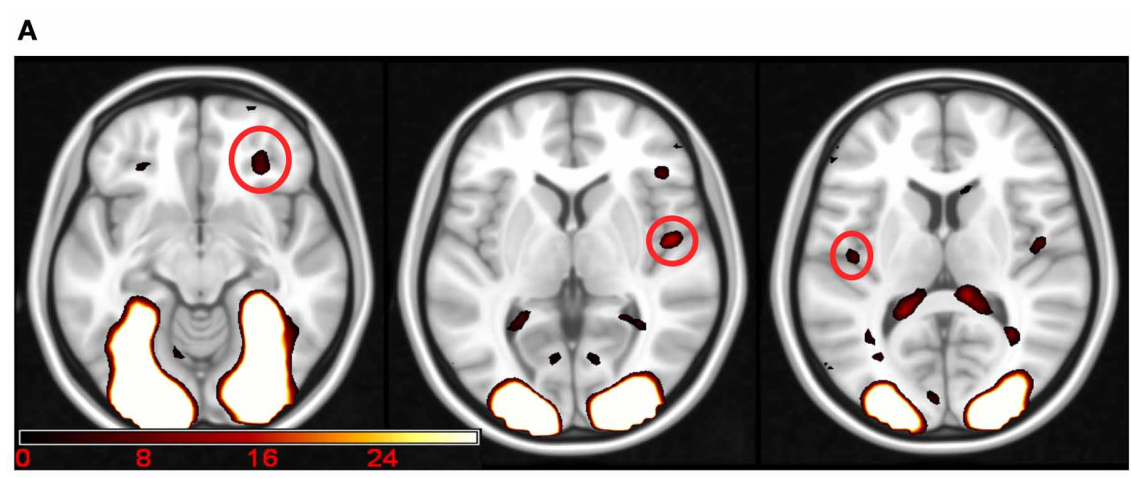

B
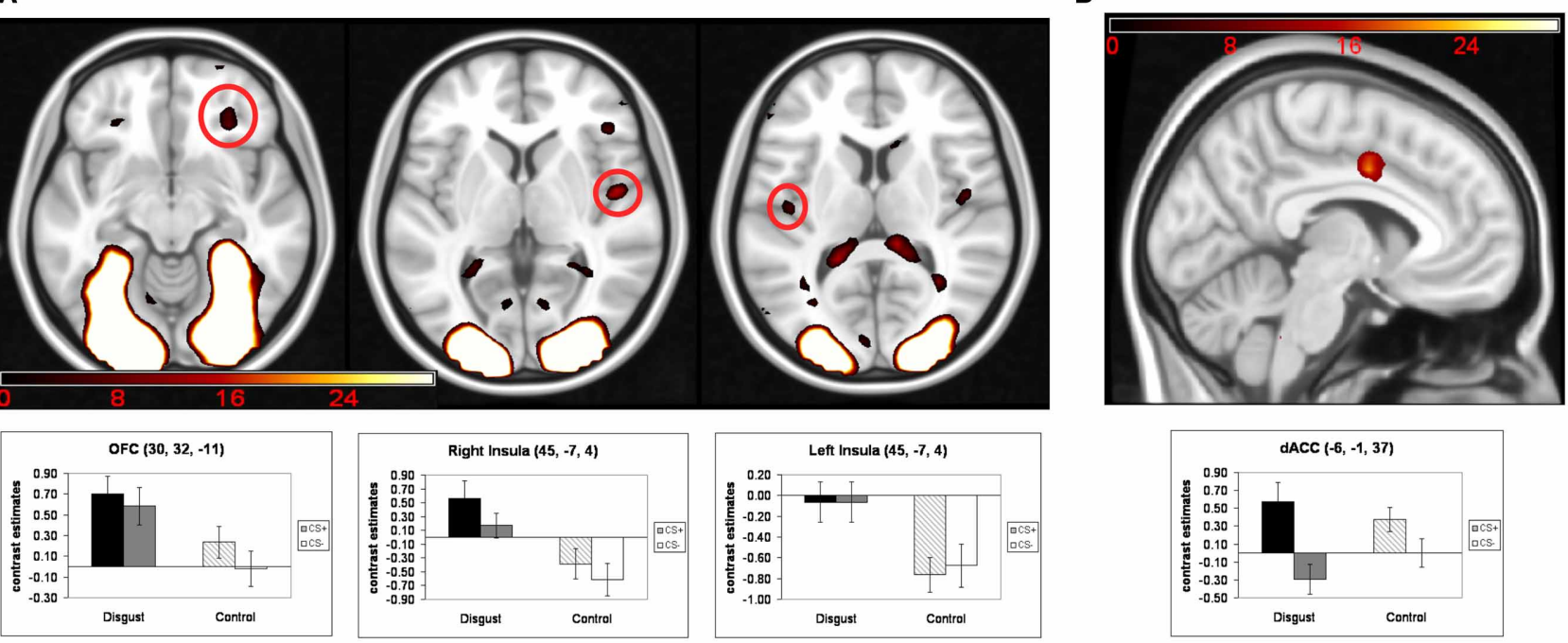

FIGURE 2 | Results of the ANOVA of conditioned responses: (A) main effects of the factor "CS-emotion"; (B) main effects of the factor "reward learning". Mean contrast estimates (and standard errors of the

mean) of the CS in the respective peak voxels are illustrated in the bar graphs. The threshold for displaying the images is set at puncorr $<0.005$ and $k>5$ voxels. 
Table 2 | Correction volume, structures, side, cluster sizes $(k)$, coordinates of peak voxels, peak $F$-values, and $p_{\text {corr }}$-values (FWE correction) for the results of the ANOVA of conditioned responses.

\begin{tabular}{|c|c|c|c|c|c|c|c|c|c|}
\hline Contrast & Correction method & Structure & Side & $\boldsymbol{k}$ & $x$ & $y$ & $z$ & $F_{\max }$ & $p_{\text {corr }}$ \\
\hline & ROI & Insula & $\mathrm{L}$ & 2 & -42 & -13 & 10 & 15.17 & 0.027 \\
\hline & whole-brain & Occipital pole & $\mathrm{L}$ & 1296 & -15 & -97 & -5 & 271.66 & $<0.001$ \\
\hline & whole-brain & Occipital pole & $\mathrm{R}$ & 1291 & 18 & -94 & 1 & 149.59 & $<0.001$ \\
\hline & whole-brain & Lingual gyrus & $\mathrm{L}$ & 12 & -6 & -88 & -19 & 29.99 & 0.012 \\
\hline Main effect of reward learning & $\mathrm{ROI}$ & $\mathrm{dACC}$ & $L$ & 23 & -6 & -1 & 37 & 20.94 & 0.005 \\
\hline Interaction CS-emotion $\times$ reward learning & \multicolumn{9}{|l|}{ No significant effects } \\
\hline
\end{tabular}

Labeling of the results from the exploratory whole-brain analyses were performed using the probabilistic Harvard-Oxford Cortical and Subcortical Atlases. The significance threshold was $p_{\text {corr }}<0.05$ (FWE-corrected). The cluster forming threshold was $p_{\text {corr }}<0.05$ and $k \geq 5$ voxel for the whole-brain analyses and puncorr $<0.001$ and $k \geq 5$ for the ROI analyses. All coordinates are given in MNI space.

\section{A}
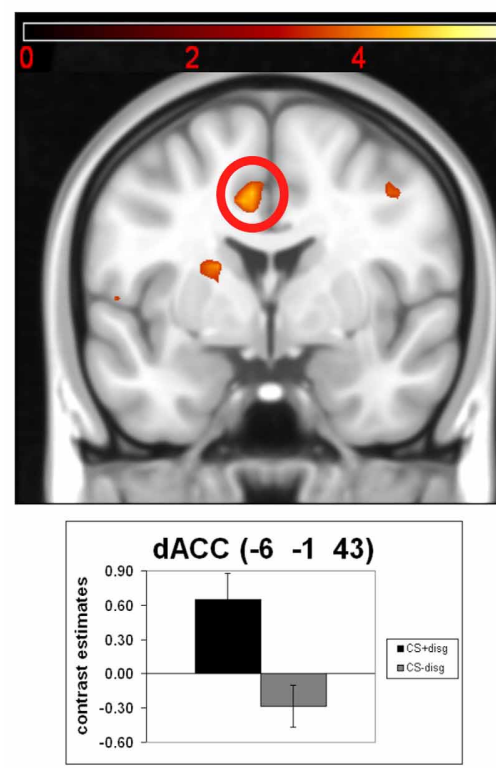
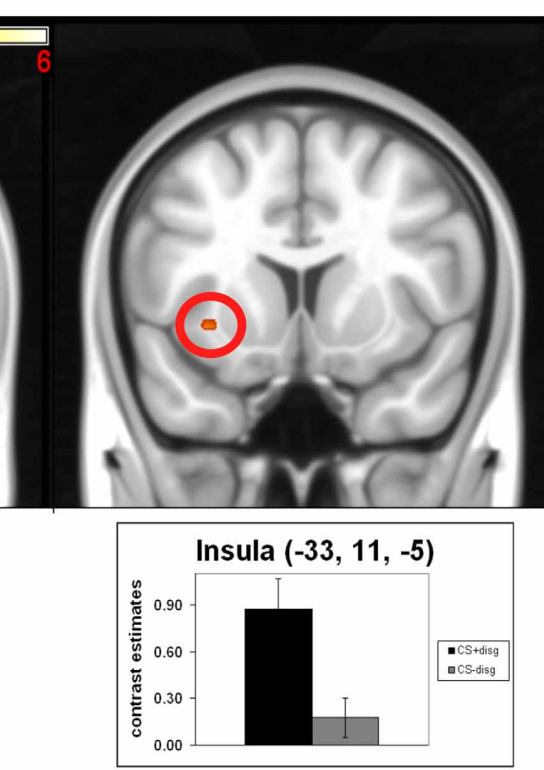

B
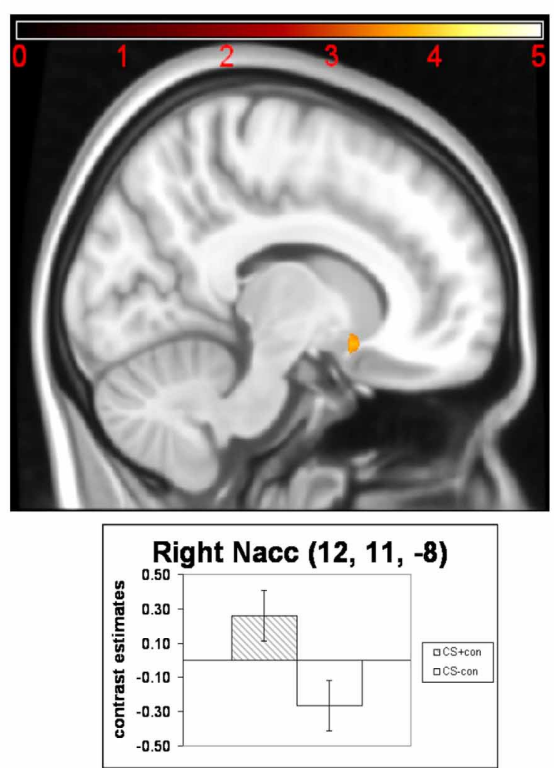

FIGURE 3 |Results of the paired $t$-tests of the separate analysis of conditioned responses in the counterconditioning and the control conditions: (A) neuronal activations for the contrast $\mathrm{CS}+{ }_{\text {disg }}$ minus CS $-_{\text {disg; }}$ (B) neuronal activations for the contrast $\mathrm{CS}_{+_{\text {con }}}$ minus

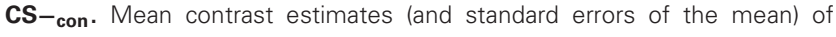
the CS in the respective peak voxels are illustrated in the bar graphs. The threshold for displaying the images is set at $p_{\text {uncorr }}<0.005$ and $k>5$ voxels.
Regarding the hemodynamic responses, analyses of variance revealed significant main effects of reward learning and anticipation as well as a main effect of the emotional content of the CS pictures. In detail, higher responses to the two CS+ were found in the AACC and stronger insula and OFC activity was observed in response to the disgust as compared to the control pictures. Again, no interaction effect (i.e., reward learning $\mathrm{x}$ CS-emotion) was observed. Taken together, the results suggest that affective processing of disgust stimuli and reward learning and anticipation may not influence each other. However, subsequent analyses revealed higher insula and dACC activity in the contrast $\mathrm{CS}+_{\text {disg }}>\mathrm{CS}-_{\text {disg }}$ implicating a potential role of these structures in the counterconditioning of disgust responses.

In line with previous findings (Eifert et al., 1988; Kerkhof et al., 2011), analyses of subjective ratings revealed conditioning effects for the valence ratings as indicated by the significant phase $\times$ CS-type interaction. Although the emotional content of the CS clearly led to highly significant overall differences between the neutral and the disgust pictures on each of the three rating dimensions, it did not differentially influence conditioning of the disgust and the control condition. Interestingly, in contrast to the valence ratings, no differential changes in subjective disgust ratings were observed between $\mathrm{CS}+_{\text {disg }}$ and disgust $\mathrm{CS}-_{\text {disg. }}$. 
This result implies that although the $\mathrm{CS}+_{\text {disg }}$ was evaluated more pleasantly than the disgust $\mathrm{CS}-$, its disgust-inducing properties were not subject to any changes. This dissociation of the two rating dimensions raises some interesting implications. First, it corresponds to the finding that on the subjective level disgust responses are very resistant to extinction (Rozin and Fallon, 1987; Smits et al., 2002), even when induced through second-order conditioning (Olatunji, 2006; Klucken et al., 2012a). On the other hand, the dissociation of the two rating dimensions disgust and valence may point to an affective conflict created through the negative affect of the disgust pictures and the positive anticipatory affect of the appetitive UCS. Nevertheless, it has to be noted that an overall decline in subjective ratings was observed, which was most likely due to habituation.

Concerning hemodynamic activity, the comparison of the disgust with the control pictures during the habituation phase as well as the main effect of CS-emotion in the analysis of conditioned responses showed stronger activity to the disgust pictures in the insula, the OFC, the amygdala, the thalamus, and the extended occipital cortex. These findings are well in line with previous studies on disgust perception and processing (for review see Cisler et al., 2009; Olatunji et al., 2010), in which altered hemodynamic activity of the insula, the OFC, the amygdala, and the occipital cortex in response to disgust-relevant stimuli have been repeatedly reported (e.g., Schienle et al., 2002b; Wright et al., 2004; Caseras et al., 2007; Jabbi et al., 2008; Schäfer et al., 2009). Underlining the importance of the insula for disgust processing, a recent meta-analysis found that the insula reliably differentiated disgust from all other emotional states (Vytal and Hamann, 2010). Activity in the OFC in response to disgust stimuli has been reported to correlate with the trait disgust sensitivity (Schienle et al., 2006; Schäfer et al., 2009), although this finding could not be replicated in the present study. Moreover, OFC activity has been found to be higher in response to contamination-related as compared to mutilation-related disgust stimuli, which is in line with our findings (Schienle et al., 2006).

In addition to the main effect of CS-emotion, we observed a main effect of reward learning and anticipation in the dACC. Stronger responses to the CS+ as compared to the corresponding CS- were observed in both conditions. Activity in the dACC is a commonly observed result in tasks that involve cues that signal an affective outcome (Martin-Soelch et al., 2007; Sehlmeyer et al., 2009). Our finding suggests that dACC activity was not affected by the emotional properties of the disgust CS. At first glance, this result is in contrast to the consistent finding of dACC activity in tasks that involve conflict or other kinds of error processing, including emotional conflict (Botvinick, 2007; Taylor et al., 2007). However, alternative views on $\mathrm{dACC}$ function propose that the dACC activity triggered by the enhanced cognitive load during conflict processing may act as a learning signal conveying adaptive control (Botvinick, 2007; Shackman et al., 2011). Moreover, the result is in line with the wealth of evidence that links the dACC to reward learning and anticipation (Martin-Soelch et al., 2007; Haber and Knutson, 2010). It also fits to the view that the dACC codes for the anticipated reward value considering that $\mathrm{CS}+{ }_{\text {disg }}$ as well as control CS $+_{\text {con }}$ predicted the same UCS (O'Doherty, 2004).
Although we observed effects of reward learning and anticipation in both conditions, the employed counterconditioning procedure did not lead to significant differences between the disgust and the control CS in areas associated with the processing of reward learning and anticipation and/or with disgust processing. This pattern of results was observed in the hemodynamic as well as the subjective responses. This result may imply that the negative affect generated by the disgust pictures and the positive affect generated by the anticipation of the monetary UCS are processed separately in the brain and the conditioned responses were based on specific visual features of the stimuli. In the subsequent analyses, enhanced dACC and insula activity to the $\mathrm{CS}+{ }_{\text {disg }}$ as compared to the $\mathrm{CS}-$ disg $_{\text {was }}$ observed. This points to potential roles for these structures in the alteration of disgust responses through counterconditioning. At least, the findings reflect the increased salience that the $\mathrm{CS}+{ }_{\text {disg }}$ obtained through the counterconditioning procedure (cf. Menon and Uddin, 2010).

Contrary to our expectations, we did not find effects of the counterconditioning procedure in NAcc and amygdala (i.e., main or interaction effects). Regarding the NAcc, we observed a differentiation in the analysis of the control condition only (i.e., in the contrast $\left.\mathrm{CS}+_{\text {con }}>\mathrm{CS}-{ }_{\text {con }}\right)$. This result is in accordance with the well-documented role of the NAcc in reward learning and anticipation (e.g., Kirsch et al., 2003 see Martin-Soelch et al., 2007; Haber and Knutson, 2010). The absence of differential NAcc activity in the contrast $\mathrm{CS}+{ }_{\text {disg }}>\mathrm{CS}-$ disg $_{\text {may indicate an influ- }}$ ence of CS valence on hemodynamic activity in this brain area, which may have slowed down the learning rate of the prediction error signal that has been associated with NAcc activity in the past (Schultz, 1997, 2002; McClure et al., 2003; O'Doherty et al., 2003). However, this assumption remains speculative and must be treated with caution. In addition, other than expected, we also did not find amygdala effects. This could be due to habituation (cf. LaBar et al., 1998) or ceiling effects of the negative pictures. Still, studies investigating reward learning and anticipation have only rarely reported effects of the amygdala (Martin-Soelch et al., 2007).

Taken together, the observed results allow the interpretation that the employed counterconditioning procedure did not directly affect disgust responding, as indexed by the lack of change in disgust ratings and the absence of interaction effects in brain regions associated to disgust responding, such as the OFC and the insula. Previous studies have demonstrated that disgust responses can be reduced through exposure (e.g., Smits et al., 2002; Olatunji et al., 2012; Viar-Paxton and Olatunji, 2012), which was also observed in this study. However, this (within-session) reduction is context dependent (Viar-Paxton and Olatunji, 2012) and remarkably smaller compared to reductions in fear responses through exposure (Smits et al., 2002; Olatunji et al., 2012). Moreover, subjective and neuronal disgust responses remain relatively stable between sessions, indicating little between-session reduction of disgust responding (Stark et al., 2004). These findings are paralleled by reports on prolonged extinction of disgustrelevant conditioned responses (Olatunji et al., 2007; Klucken et al., 2013). Thus, the lack of change in subjective and hemodynamic disgust responding in this study is in line with the view that disgust responses are particularly difficult to modify 
(Rozin and Fallon, 1987; Olatunji et al., 2010; Mason and Richardson, 2012). Furthermore, specific stimulus attributes, such as the nature and the similarity of the disgust stimuli (i.e., faeces vs. other kinds of disgust stimuli, e.g., rotten bodies, cockroaches, cf. Rozin and Fallon, 1987) may have also influenced the observed results in the current study. Nevertheless, the lack of effects in disgust responding does not necessarily imply that the disgust responses were totally unaffected by the counterconditioning procedure. In the case of fear, it has been demonstrated that the level of fear during exposure is not predictive of the level of fear at re-exposure (see review by Craske et al., 2008). Moreover, it has been shown that the valence difference between CS+ and CS- is predictive for the amount of behavioral reinstatement in a later test phase in a fear conditioning experiment (Dirikx et al., 2004, 2007). However, it is an open question whether these findings hold for the counterconditioning of disgust responses.

We would finally like to address some potential shortcomings of the present study. First, the observed effects may only hold for disgust stimuli from the category contamination / body secretion (cf. Rozin and Fallon, 1987). It is unclear whether other types of disgust stimuli could have led to different results. Second, it is possible that a closer fit of CS and UCS could lead to stronger effects of the counterconditioning procedure, for example the usage of pleasant odor as UCS. In addition, we cannot

\section{REFERENCES}

Barcikowski, R. S., and Robey, R. R. (1984). Decisions in single group repeated measures analysis: statistical tests and three computer packages. Am. Stat. 38, 148-150.

Boik, R. J. (1981). A priori tests in repeated measures designs: effects of nonsphericity. Psychometrika 46, 241-255. doi: 10.1007/ BF02293733

Botvinick, M. M. (2007). Conflict monitoring and decision making: reconciling two perspectives on anterior cingulate function. Cogn. Affect. Behav. Neurosci. 7, 356-366. doi: 10.3758/CABN.7.4.356

Bouton, M. E. (2004). Context and behavioral processes in extinction. Learn. Mem. 11, 485-494. doi: 10.1101/lm.78804

Calder, A. J., Lawrence, A. D., and Young, A. W. (2001). Neuropsychology of fear and loathing. Nat. Rev. Neurosci. 2, 352-363. doi: 10.1038/35072584

Carter, C. S., and van Veen, V. (2007). Anterior cingulate cortex and conflict detection: an update of theory and data. Cogn. Affect. Behav. Neurosci. 7, 367-379. doi: 10.3758/CABN.7.4.367

Caseras, X., Mataix-Cols, D., An, S. K., Lawrence, N. S., Speckens, A., Giampietro, V., et al. (2007). Sex differences in neural responses to disgusting visual stimuli: implications for disgust-related psychiatric disorders. Biol. Psychiatry 62, 464-471. doi: 10.1016/j.biopsych.2006.10.030

Cisler, J. M., Olatunji, B. O., and Lohr, J. M. (2009). Disgust, fear, and the anxiety disorders. A critical review. Clin. Psychol. Rev. 29, 34-46. doi: 10.1016/j.cpr.2008.09.007

Cox, S. M., Andrade, A., and Johnsrude, I. S. (2005). Learning to like: a role for human orbitofrontal cortex in conditioned reward. J. Neurosci. 25, 2733-2740. doi: 10.1523/JNEUROSCI.3360-04.2005

Craske, M. G., Kircanski, K., Zelikowsky, M., Mystkowski, J., Chowdhury, N., and Baker, A. (2008). Optimizing inhibitory learning during exposure therapy. Behav. Res. Ther. 46, 5-27. doi: 10.1016/j.brat.2007.10.003

Daw, N. D., Kakade, S., and Dayan, P. (2002). Opponent interactions between serotonin and dopamine. Neural Netw. 15, 603-616. doi: 10.1016/S0893-6080(02)00052-7

Deichmann, R., Gottfried, J. A., Hutton, C., and Turner, R. (2003). Optimized EPI for fMRI studies of the orbitofrontal cortex. Neuroimage 19, 430-441. doi: 10.1016/S1053-8119(03)00073-9

Dickinson, A., and Pearce, J. M. (1977). Inhibitory interactions between appetitive and aversive stimuli. Psychol. Bull. 84, 690-711. doi: 10.1037/0033-2909.84.4.690

exclude that conditioning was influenced by the repeated presentation and the relatively long presentation time of the stimuli, which clearly created habituation effects. Finally, since our study investigated healthy subjects, it is unclear how exaggerated disgust responses in subjects suffering from psychiatric disorders are affected by counterconditioning.

In conclusion, the observed effects in the behavioral and the fMRI data suggest that the emotional content of the disgust pictures did not differentially alter the magnitude of the conditioned responses. The results imply that disgust responses and reward learning and anticipation may not influence each other. Nevertheless, the separate analysis of the counterconditioning condition indicates that the dACC and the insula may play a role in the alteration of disgust responses through counterconditioning. In sum, the results of this first study on the neuronal correlates of counterconditioning in humans add to the ongoing debate on the transfer of neuronal foundations of emotional learning processes to behavioral treatment strategies and add to a more sophisticated understanding of human emotions.

\section{ACKNOWLEDGMENTS}

This work was supported by the DFG Graduiertenkolleg GRK 885 "NeuroAct". We would like to thank Dr. Carlo Blecker, Luise Blochberger and Csilla Fodor for assistance with data collection and Dr. Andrea Hermann for helpful discussions on this project.

Dirikx, T., Hermans, D., Vansteenwegen, D., Baeyens, F., and Eelen, P. (2004). Reinstatement of extinguished conditioned responses and negative stimulus valence as a pathway to return of fear in humans. Learn. Mem. 11, 549-554. doi: 10.1101/lm.78004

Dirikx, T., Hermans, D., Vansteenwegen, D., Baeyens, F., and Eelen, P. (2007). Reinstatement of conditioned responses in human differential fear conditioning. J. Behav. Ther. Exp. Psychiatry 38, 237-251. doi: 10.1016/j.jbtep.2006.04.001

Eifert, G. H., Craill, L., Carey, E., and O'Connor, C. (1988). Affect modification through evaluative conditioning with music. Behav. Res. Ther. 26, 321-330. doi: 10.1016/0005-7967(88)90084-8

Fitzgerald, D. A., Posse, S., Moore, G. J., Tancer, M. E., Nathan, P. J., and Phan, K. L. (2004). Neural correlates of internally-generated disgust via autobiographical recall: a functional magnetic resonance imaging investigation. Neurosci. Lett. 370, 91-96. doi: 10.1016/j.neulet.2004.08.007

Gottfried, J. A., O’Doherty, J. P., and Dolan, R. J. (2002). Appetitive and aversive olfactory learning in humans studied using event-related functional magnetic resonance imaging. J. Neurosci. 22, 10829-10837.
Haber, S. N., and Knutson, B. (2010). The reward circuit: linking primate anatomy and human imaging. Neuropsychopharmacology 35, 4-26. doi: 10.1038/npp.2009.129

Haidt, J., McCauley, C., and Rozin, P. (1994). Individual differences in sensitivity to disgust: A scale sampling seven domains of disgust elicitors. Pers. Indiv. Differ. 16, 701-713. doi: 10.1016/0191-8869(94)90212-7

Hubert, M., and van der Veeken, S. (2008). Outlier detection for skewed data. J. Chemometrics 22, 235-246. doi: 10.1002/cem.1123

Jabbi, M., Bastiaansen, J., Keysers, C., and Lauwereyns, J. (2008). A common anterior insula representation of disgust observation, experience and imagination shows divergent functional connectivity pathways. PLoS ONE 3:e2939. doi: 10.1371/journal.pone.0002939

Jong, P. J. de, Vorage, I., and van den Hout, M. A. (2000). Counterconditioning in the treatment of spider phobia: effects on disgust, fear and valence. Behav. Res. Ther. 38, 1055-1069. doi: 10.1016/S0005-7967(99)00135-7

Kerkhof, I., Vansteenwegen, D., Baeyens, F., and Hermans, D. (2011). Counterconditioning. An effective technique for changing conditioned preferences. Exp. Psychol. 58, 31-38. doi: 10.1027/1618-3169/a000063 
Kirsch, P., Schienle, A., Stark, R., Sammer, G., Blecker, C., Walter, B., et al. (2003). Anticipation of reward in a nonaversive differential conditioning paradigm and the brain reward system: an eventrelated fMRI study. Neuroimage 20, 1086-1095. doi: 10.1016/S10538119(03)00381-1

Klucken, T., Kagerer, S., Schweckendiek, J., Tabbert, K., Vaitl, D., and Stark, R. (2009a). Neural, electrodermal and behavioral response patterns in contingency aware and unaware subjects during a picturepicture conditioning paradigm. Neuroscience 158, 721-731. doi: 10.1016/j.neuroscience.2008.09.049

Klucken, T., Schweckendiek, J., Merz, C. J., Tabbert, K., Walter, B., Kagerer, S., et al. (2009b). Neural activations of the acquisition of conditioned sexual arousal: effects of contingency awareness and sex. J. Sex. Med. 6, 3071-3085. doi: 10.1111/j.1743-6109.2009.01405.x

Klucken, T., Schweckendiek, J., Koppe, G., Merz, C., Kagerer, S., Walter, B., et al. (2012a). Neural correlates of disgustand fear-conditioned responses. Neuroscience 201, 209-218. doi: 10.1016/j.neuroscience.2011.11.007

Klucken, T., Wehrum, S., Schweckendiek, J., Merz, C. J., Hennig, J., Vaitl, D., et al. (2012b). The 5-HTTLPR polymorphism is associated with altered hemodynamic responses during appetitive conditioning. Hum. Brain Mapp. doi: 10.1002/hbm.22085. [Epub ahead of print].

Klucken, T., Schweckendiek, J., Merz, C. J., Vaitl, D., and Stark, R. (2013). Dissociation of neuronal, electrodermal, and evaluative responses in disgust extinction. Behav. Neurosci. doi: 10.1037/a0032331. [Epub ahead of print].

LaBar, K. S., Gatenby, J. C., Gore, J. C., LeDoux, J. E., and Phelps, E. A. (1998). Human amygdala activation during conditioned fear acquisition and extinction: a mixed-trial fMRI study. Neuron 20, 937-945. doi: 10.1016/S08966273(00)80475-4

Lang, P. J., Bradley, M. M., and Cuthbert, B. N. (2008). International Affective Picture System (IAPS): Affective Ratings of Pictures and Instruction Manual. Technical Report A-8. Gainesville, FL: University of Florida.

Martin-Soelch, C., Linthicum, J., and Ernst, M. (2007). Appetitive conditioning: neural bases and implications for psychopathology. Neurosci.
Biobehav. Rev. 31, 426-440. doi: 10.1016/j.neubiorev.2006.11.002

Mason, E. C., and Richardson, R. (2012). Treating disgust in anxiety disorders. Clin. Psychol.Sci. Pract. 19, 180-194.

McClure, S. M., Berns, G. S., and Montague, P. R. (2003). Temporal prediction errors in a passive learning task activate human striatum. Neuron 38, 339-346. doi: 10.1016/S0896-6273(03)00154-5

McNally, R. J. (2007). Mechanisms of exposure therapy: How neuroscience can improve psychological treatments for anxiety disorders. Clin. Psychol. Rev. 27, 750-759. doi: 10.1016/j.cpr.2007. 01.003

Menon, V., and Uddin, L. Q. (2010). Saliency, switching, attention and control: a network model of insula function. Brain Struct. Funct. 214, 655-667. doi: 10.1007/s00429-0100262-0

Merz, C. J., Tabbert, K., Schweckendiek, J., Klucken, T., Vaitl, D., Stark, R., et al. (2010). Investigating the impact of sex and cortisol on implicit fear conditioning with fMRI. Psychoneuroendocrinology 35, 33-46. doi: 10.1016/ j.psyneuen.2009.07.009

O’Doherty, J. P. (2004). Reward representations and reward-related learning in the human brain: insights from neuroimaging. Curr. Opin. Neurobiol. 14, 769-776. doi: 10.1016/j.conb.2004.10.016

O’Doherty, J. P., Dayan, P., Friston, K., Critchley, H., and Dolan, R. J. (2003). Temporal difference models and reward-related learning in the human brain. Neuron 38, 329-337. doi: 10.1016/S08966273(03)00169-7

Olatunji, B. O. (2006). Evaluative learning and emotional responding to fearful and disgusting stimuli in spider phobia. J. Anxiety Disord. 20, 858-876. doi: 10.1016/j.janxdis.2006.01.005

Olatunji, B. O., Ciesielski, B. G., Wolitzky-Taylor, K. B., Wentworth, B. J., and Viar, M. A. (2012). Effects of experienced disgust on habituation during repeated exposure to threat-relevant stimuli in blood-injection-injury phobia. Behav. Ther. 43, 132-141. doi: 10.1016/j.beth.2011.04.002

Olatunji, B. O., Cisler, J., McKay, D., and Phillips, M. L. (2010). Is disgust associated with psychopathology. Emerging research in the anxiety disorders. Psychiatry Res. 175, 1-10. doi: 10.1016/j.psychres.2009.04.007

Olatunji, B. O., Forsyth, J. P., and Cherian, A. (2007). Evaluative differential conditioning of disgust: a sticky form of relational learning that is resistant to extinction. J. Anxiety Disord. 21, 820-834. doi: 10.1016/j.janxdis.2006.11.004

Penny, W., and Henson, R. N. A. (2007). "Analysis of variance," in Statistical Parametric Mapping. The Analysis of Functional Brain Images, eds K. J. Friston, J. T. Ashburner, S. J. Kiebel, T. E. Nichols, and W. D. Penny (Amsterdam: Elsevier Academic Press), 166-178.

Phan, K. L., Wager, T. D., Taylor, S. F., and Liberzon, I. (2004). Functional neuroimaging studies of human emotions. CNS Spectr. 9, 258-266.

Raes, A., de Raedt, R., Fias, W., Koster, E., and van Damme, S. (2009). Does contingency awareness mediate the influence of emotional learning on the cueing of visual attention. Psychol. Res. 73, 107-113. doi: 10.1007/s00426-0080141-y

Rozin, P., and Fallon, A. E. (1987) A perspective on disgust. Psychol. Rev. 94, 23-41. doi: 10.1037/0033295X.94.1.23

Schäfer, A., Leutgeb, V., Reishofer, G., Ebner, F., and Schienle, A. (2009). Propensity and sensitivity measures of fear and disgust are differentially related to emotion-specific brain activation. Neurosci. Lett. 465, 262-266. doi 10.1016/j.neulet.2009.09.030

Schienle, A., Alter, B., Stark, R., and Vaitl, D. (2002a). Ein Fragebogen zur Erfassung der Ekelempfindlichkeit (FEE). Z. Klin. Psychol. Psychiatr. Psychother. 31, 110-120. doi: 10.1026//1616-3443.31.2.110

Schienle, A., Stark, R., Walter, B., Blecker, C., Ott, U., Kirsch, P., et al. (2002b). The insula is not specifically involved in disgust processing: an fMRI study. Neuroreport 13 , 2023-2026. doi: 10.1097/00001756200211150-00006

Schienle, A., Schäfer, A., Hermann, A., Walter, B., Stark, R., and Vaitl, D. (2006). fMRI responses to pictures of mutilation and contamination. Neurosci. Lett. 393, 174-178. doi: 10.1016/j.neulet.2005.09.072

Schienle, A., Stark, R., Walter, B., and Vaitl, D. (2003). The connection between disgust sensitivity and blood-related fears, faintness symptoms, and obsessivecompulsiveness in a non-clinical sample. Anxiety Stress Coping 16, 185-193.

Schiller, D., Cain, C. K., Curley, N. G., Schwartz, J. S., Stern, S. A., LeDoux, J. E., et al. (2008). Evidence for recovery of fear following immediate extinction in rats and humans. Learn. Mem. 15, 394-402. doi: 10.1101/lm.909208

Schultz, W. (1997). A neural substrate of prediction and reward. Science 275, 1593-1599. doi: $10.1126 /$ science.275.5306.1593

Schultz, W. (2002). Getting formal with dopamine and reward. Neuron 36, 241-263. doi: 10.1016/S08966273(02)00967-4

Schweckendiek, J., Klucken, T. Merz, C. J., Tabbert, K., Walter, B., Ambach, W., et al. (2011). Weaving the (neuronal) web: Fear learning in spider phobia. Neuroimage 54, 681-688. doi: 10.1016/j.neuroimage.2010.07.049

Sehlmeyer, C., Schöning, S., Zwitserlood, P., Pfleiderer, B., Kircher, T., Arolt, V., et al. (2009). Human fear conditioning and extinction in neuroimaging: a systematic review. PLoS ONE 4:e5865. doi: 10.1371/journal.pone.0005865

Shackman, A. J., Salomons, T. V., Slagter, H. A., Fox, A. S., Winter, J. J., and Davidson, R. J. (2011). The integration of negative affect, pain and cognitive control in the cingulate cortex. Nat. Rev. Neurosci. 12, 154-167. doi: 10.1038/ nrn2994

Smits, J. A. J., Telch, M. J., and Randall, P. K. (2002). An examination of the decline in fear and disgust during exposure-based treatment. Behav. Res. Ther. 40, 1243-1253. doi: 10.1016/S00057967(01)00094-8

Stark, R., Schienle, A., Sarlo, M., Palomba, D., Walter, B., and Vaitl, D. (2005). Influences of disgust sensitivity on hemodynamic responses towards a disgust-inducing film clip. Int. J. Psychophysiol. 57, 61-67. doi: 10.1016/j.ijpsycho. 2005.01.010

Stark, R., Schienle, A., Walter, B., Kirsch, P., Blecker, C., Ott, U., et al. (2004). Hemodynamic effects of negative emotional pictures - a test-retest analysis. Neuropsychobiology 50, 108-118. doi: $10.1159 / 000077948$

Stark, R., Zimmermann, M., Kagerer, S., Schienle, A., Walter, B. Weygandt, M., et al. (2007). Hemodynamic brain correlates of disgust and fear ratings. Neuroimage 37, 663-673. doi: 10.1016/j.neuroimage.2007.05.005

Taylor, S. F., Stern, E. R., and Gehring, W. J. (2007). Neural systems for error monitoring: recent findings and theoretical perspectives. Neuroscientist 13, 160-172. doi: $10.1177 / 1073858406298184$ 
Viar-Paxton, M. A., and Olatunji, B. O. (2012). Context Effects on Habituation to DisgustRelevant Stimuli. Behav. Modif. 36, 705-722. doi: 10.1177/01454455 12446189

Vytal, K., and Hamann, S. (2010). Neuroimaging support for discrete neural correlates of basic emotions: a voxel-based meta-analysis. J. Cogn. Neurosci. 22, 2864-2885. doi: 10.1162/jocn.2009.21366

Weiskopf, N., Hutton, C., Josephs, O., and Deichmann, R. (2006). Optimal EPI parameters for reduction of susceptibility-induced
BOLD sensitivity losses: a wholebrain analysis at $3 \mathrm{~T}$ and $1.5 \mathrm{~T}$. Neuroimage 33, 493-504. doi: 10.1016/j.neuroimage.2006.07.029

Worsley, K. J. (2007). "Random field theory," in Statistical Parametric Mapping, eds K. J. Friston, J. T. Ashburner, S. J. Kiebel, T. E. Nichols, and W. D. Penny (Amsterdam: Elsevier Academic Press), 232-237.

Wright, P., He, G., Shapira, N. A., Goodman, W. K., and Liu, Y. (2004). Disgust and the insula: fMRI responses to pictures of mutilation and contamination. Neuroreport
15, 2347-2351. doi: 10.1097/ 00001756-200410250-00009

Conflict of Interest Statement: The authors declare that the research was conducted in the absence of any commercial or financial relationships that could be construed as a potential conflict of interest.

Received: 08 April 2013; accepted: 17 June 2013; published online: 08 July 2013.

Citation: Schweckendiek J, Klucken T, Merz CJ, Kagerer S, Walter B, Vaitl
$D$ and Stark $R$ (2013) Learning to like disgust: neuronal correlates of counterconditioning. Front. Hum. Neurosci. 7:346. doi: 10.3389/fnhum. 2013.00346

Copyright (c) 2013 Schweckendiek, Klucken, Merz, Kagerer, Walter, Vaitl and Stark. This is an open-access article distributed under the terms of the Creative Commons Attribution License, which permits use, distribution and reproduction in other forums, provided the original authors and source are credited and subject to any copyright notices concerning any third-party graphics etc. 